(2) Open Access Full Text Article

\title{
Elevated creatine kinase does not necessarily correspond temporally with onset of muscle rigidity in neuroleptic malignant syndrome: a report of two cases
}

\author{
This article was published in the following Dove Press journal: \\ Neuropsychiatric Disease and Treatment \\ 12 December 2012 \\ Number of times this article has been viewed
}

\author{
Koichi Nisijima \\ Department of Psychiatry, Jichi \\ Medical University, Tochigi, Japan
}

Correspondence: Koichi Nisijima Department of Psychiatry, Yakushiji Shimotuke-Shi Tochigi-Ken,

329-0498, Japan

Tel +81285587364

Fax +81285446198

Email midorin@jichi.ac.jp

\begin{abstract}
Neuroleptic malignant syndrome is an uncommon but dangerous complication of antipsychotic drugs, characterized by clinical symptoms that include hyperthermia, severe muscle rigidity, autonomic dysfunction, and altered mental state. Serum creatine kinase (CK) elevation occurs in over $90 \%$ of cases. Many diagnostic criteria sets for neuroleptic malignant syndrome have been proposed, all of which include hyperthermia and muscle rigidity as major symptoms, and serum CK elevation as either a major or minor symptom. In general, elevated CK occurs in the initial stage of neuroleptic malignant syndrome and corresponds temporally with the onset of muscle rigidity. However, in some exceptional cases, CK elevation and emergence of muscle rigidity do not appear in the same stage, making early diagnosis of neuroleptic malignant syndrome more difficult. Two rare cases of neuroleptic malignant syndrome are presented in which elevated serum CK and emergence of muscle rigidity did not occur in the same stage of neuroleptic malignant syndrome. An elevated CK level is common in the early stage of neuroleptic malignant syndrome, suggesting that serum CK elevation is a useful indicator for early detection of neuroleptic malignant syndrome. However, a definitive diagnosis of neuroleptic malignant syndrome must be determined from the presence of specific clinical symptoms.

Keywords: neuroleptic malignant syndrome, creatine kinase, muscle rigidity
\end{abstract}

\section{Introduction}

Neuroleptic malignant syndrome (NMS) is a serious adverse reaction to antipsychotic drugs. The syndrome is characterized by four main symptoms, ie, hyperthermia, extreme muscle rigidity, autonomic instability, and mental status changes. The first diagnostic criteria proposed for NMS by Levenson in $1985^{1}$ included major criteria (fever, rigidity, and elevated creatine kinase [CK] level) and minor criteria (tachycardia, abnormal blood pressure, tachypnea, altered level of consciousness, diaphoresis, and leukocytosis). Diagnosis using this criteria set rests on the presence of all three major criteria or two major criteria and four minor criteria.

Other diagnostic standards for NMS have since been proposed, ${ }^{2-4}$ and all include hyperthermia and muscle rigidity as major symptoms and elevated serum $\mathrm{CK}$ as a minor symptom. Serum CK elevation is not specific to $\mathrm{NMS},{ }^{5}$ but is reported to be found in over $90 \%$ of patients with NMS. ${ }^{3,6}$ Therefore, an elevated CK level is suggestive of NMS. In addition, follow-up of the CK level has been reported to be helpful in establishing the rate of amelioration of NMS. ${ }^{7}$ 
Although the literature contains numerous case reports on NMS, there have been no detailed studies of the relationship between level of serum CK and degree of muscle rigidity following onset of NMS. Rosebush and Stewart ${ }^{8}$ reported that the CK level peaked on days 2 and 3 after onset in $64 \%$ of 24 consecutive cases, and Velamoor et $\mathrm{al}^{9}$ pointed out muscle rigidity and mental status changes as the initial manifestations in $82.3 \%$ of 222 previously reported cases. The findings of these two studies suggest that an increased serum CK level and muscle rigidity both occur in the early stage of NMS.

We report here two cases in which muscle rigidity did not emerge with elevated CK in the early stage of NMS, but rather emerged after the serum CK level had been normalized mid stage. These cases suggest that serum CK elevation does not necessarily parallel the degree of muscle rigidity in patients with NMS. In such cases, diagnosis of NMS may be delayed or the severity of NMS may be incorrectly evaluated.

\section{Case report I}

A 32-year-old man who had been suffering from schizophrenia for 2 years was admitted to the hospital for worsening of his mental condition, including psychomotor agitation, auditory hallucinations, and sleeplessness. On admission, he was prescribed chlorpromazine $100 \mathrm{mg}$ /day. On hospital day 10 , the dose of chlorpromazine was increased to $150 \mathrm{mg}$ and haloperidol $9 \mathrm{mg}$ /day was added because the excitation had not improved. On hospital day 13, chlorpromazine $50 \mathrm{mg}$ was given intramuscularly to alleviate the excitation. The patient was drowsy throughout hospital day 14 , and his body temperature increased to $39.7^{\circ} \mathrm{C}$ and heart rate to 120 beats per minute on hospital day 15 (day 1 of NMS). The results of blood tests on hospital day 16 revealed an elevated serum CK of 540 (normal range 46-210) IU/L and a high white blood count of $11,500 / \mathrm{mm}^{3}$. Brain computed tomography, a cerebrospinal fluid study, and a thyroid function test revealed no abnormalities. No obvious infection was detected. Therefore, NMS was suspected. However, because muscle rigidity was not found, neuroleptics were continued. On hospital day 17, serum CK was increased to $722 \mathrm{IU} / \mathrm{L}$. The patient continued to be restless and began to cry in a loud voice. Administration of an intravenous bolus of haloperidol $10 \mathrm{mg}$ was started on hospital day 21 . On hospital day 23 , the patient was unresponsive, but intravenous administration of haloperidol was continued because serum CK had normalized to $143 \mathrm{IU} / \mathrm{L}$. Generalized muscular rigidity was noted on hospital day 24 (day 10 of NMS).
Symptoms of autonomic disturbance started to appear (mydriasis, generalized perspiration, and diarrhea), and NMS was finally diagnosed on hospital day 26. Intravenous injections of haloperidol were stopped, and fluid replacement and dantrolene $100 \mathrm{mg}$ /day were started. Serum CK remained in the normal range (79 IU/L). On hospital day 27, serum CK was measured again and was still in the normal range (83 IU/L). On hospital day 29, the akinetic mutism was improved, and on hospital day 32 , fever, muscular rigidity, diaphoresis, and diarrhea were no longer present, and it was judged that the patient no longer had NMS (day 20 from onset of symptoms).

To monitor changes in muscle rigidity and serum CK values more accurately, the NMS evaluation scale proposed by Sachdev ${ }^{10}$ was used. Muscle rigidity was rated as follows: 0 , nil (no rigidity); 1, mild (slight rigidity present, particularly obvious on recruitment of muscles with jaw clenching); 2, moderate (definitely present to a significant degree but produces no limitation of passive movement); or 3, severe (rigidity that produces some limitation of passive movement). The changes in these values during the course of NMS were monitored, and changes in serum CK levels and body temperature were also assessed. These data are shown in Figure 1A.

\section{Case report 2}

A 29-year-old woman with mild mental retardation and bipolar disorder was admitted to the hospital for emotional instability and dysthymia. She engaged in acts of self-mutilation, such as attempting to cut her hand with a knife. Her mood was unstable for the 6 months following admission. Paliperidone $6 \mathrm{mg} /$ day, chlorpromazine $150 \mathrm{mg} /$ day, and carbamazepine $300 \mathrm{mg} /$ day were prescribed. Eight months later, the dysthymia had stabilized and the patient was scheduled for discharge. However, around hospital day 240, the patient lost vigor and lay in bed most of the time. On hospital 247, fever (body temperature, $38.3^{\circ} \mathrm{C}$ ) was noted (day 1 of NMS) and this fever of unknown origin (body temperature approximately $37.5^{\circ} \mathrm{C}$ ) persisted. On hospital day 250 , serum CK was 4273 (normal range 46-210) IU/L and white cell count was $6400 / \mathrm{mm}^{3}$. Results of other laboratory tests and brain imaging were normal. There was no sign of muscular rigidity or dysphagia, but extreme diaphoresis appeared. Based on the CK level, NMS was suspected. All antipsychotics were stopped, and fluid replacement and dantrolene $75 \mathrm{mg}$ /day were started.

On hospital day 253, the serum CK value was $841 \mathrm{IU} / \mathrm{L}$. In the evening of the same day, the patient became nauseous 
A

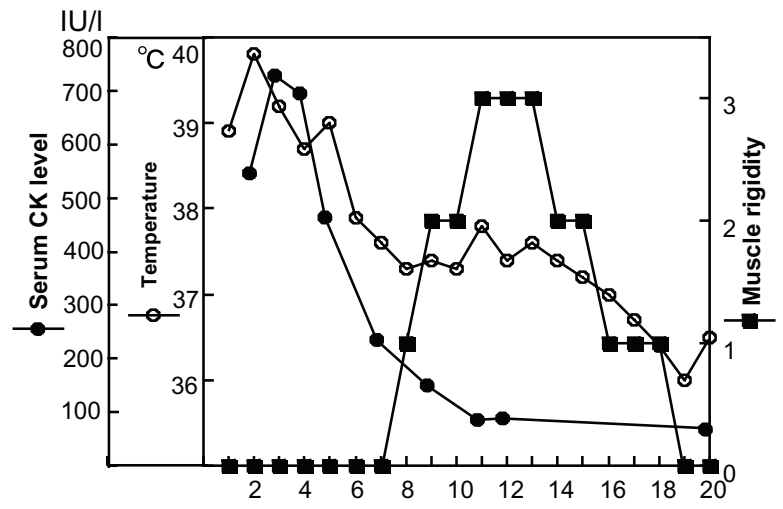

B

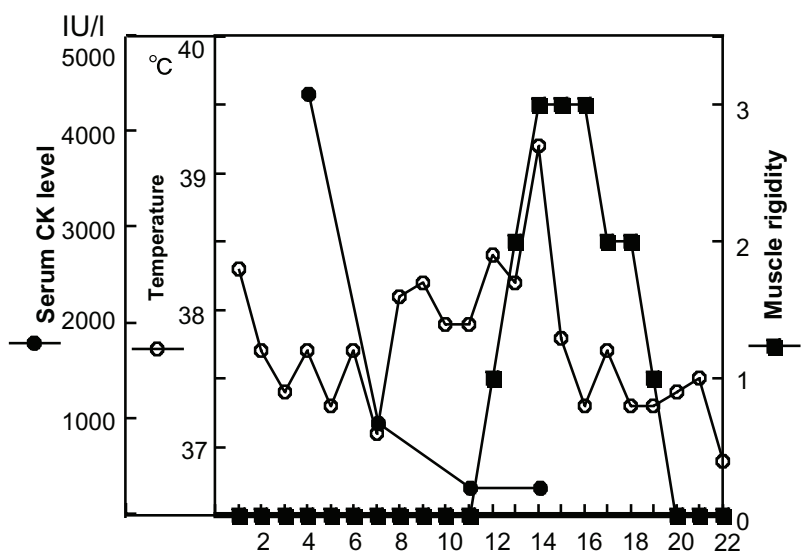

Figure I Changes in serum creatine kinase level, muscle rigidity, and body temperature in case I (A) and case 2 (B).

Notes: Both patients with neuroleptic malignant syndrome showed nonsimultaneous occurrence of elevated serum creatine kinase $(\bullet)$ and onset of muscle rigidity (•).

and vomited. Because the physician in charge was not present, a resident administered metoclopramide $10 \mathrm{mg}$ intravenously (day 7 of NMS). On hospital day 254, her body temperature was $38.1^{\circ} \mathrm{C}$ and tremor was noted in both upper limbs. In addition, the patient was almost mute. However, muscle rigidity was not observed. Serum CK normalized to $154 \mathrm{IU} / \mathrm{L}$ on hospital day 257, and dantrolene was stopped on hospital days 258 and 259 when it was judged that the NMS had improved. On hospital day 260, although her serum CK value was $160 \mathrm{IU} / \mathrm{L}$, her white cell count was high at $13,100 / \mathrm{mm}^{3}$ and body temperature was $39.2^{\circ} \mathrm{C}$. In addition, tremor and muscle rigidity was noted in all four limbs. Although serum CK was normal, it was judged that the NMS had not yet improved because full-blown NMS symptoms were evident. Dantrolene was restarted and from hospital day 264 onwards, the patient showed signs of responsiveness, such as opening her eyes when called. On hospital day 268 (day 22 of NMS), the muscle rigidity, dysphagia, tremor, and diaphoresis disappeared, and her body temperature decreased to $36^{\circ} \mathrm{C}$. Changes in muscle rigidity, serum CK level, and body temperature are shown in Figure 1B.

\section{Discussion}

Typical neuroleptics, ie, chlorpromazine and haloperidol, were administered in case 1 , and the patient presented with fever (body temperature $>38^{\circ} \mathrm{C}$ ) and elevated serum CK levels. A typical neuroleptic and an atypical neuroleptic, ie, chlorpromazine and paliperidone, respectively, were administered in case 2 and the patient presented with fever (body temperature $>38^{\circ} \mathrm{C}$ ) and elevated serum CK. These two drugs appear to have been the cause of NMS in case 2 . However, the patient was also treated with metoclopramide (on day 7 of NMS) for nausea and vomiting. Although metoclopramide is an antiemetic, it also has anti-dopamine $\mathrm{D}_{2}$ receptor effects and reports of metoclopramide-induced NMS have appeared in the literature..$^{11}$ Therefore, metoclopramide may have caused the NMS in case 2 .

In both cases, the four major clinical symptoms of NMS and elevated serum CK were present in the initial or middle stage of the illness and therefore diagnosis of NMS was not so difficult. However, the unusual timing of the characteristic symptoms did present some difficulties. The most characteristic feature of NMS was the increase in serum CK in the initial stage. However, muscle rigidity was not seen at that time, and appeared only after CK levels had decreased to the normal range. Serum CK values usually peak on day 2 to 3 of NMS as they did in both cases, but the muscle rigidity and mental status changes that are often found in the initial stage of NMS were not apparent. Védie et a ${ }^{12}$ pointed out that CK elevation occurs very early and corresponds temporally with the onset of rigidity, and while Harsch ${ }^{13}$ reported that CK elevation and fever did not necessarily parallel the duration or degree of muscle rigidity in nine cases of NMS, he did not observe an inverse relationship between elevated CK level and the appearance of rigidity.

Given these findings, the nonsimultaneous occurrence of muscle rigidity and elevated serum CK levels observed in the present cases appears to be a rare finding. In case 1 , fever and elevated serum CK were evident in the initial stage of NMS, but there was no muscle rigidity, which delayed the diagnosis of NMS. In case 2, NMS was suspected in the early stage when fever and elevated CK were found, but serum CK normalized as expected over the course of the illness, leading to the conclusion that the NMS had improved when it had not, and dantrolene administration was stopped for 2 days.

Serum CK elevation is not specific to NMS. However, it occurs in up to $90 \%$ of NMS cases ${ }^{6}$ and is found at a high 
rate in the initial stage of NMS. ${ }^{8}$ Therefore, if an increase in serum CK is found along with fever of unknown origin during the administration of neuroleptics, physicians should suspect NMS. In this respect, serum CK elevation may be a useful indicator for early detection of NMS. However, this elevation of accompanying fever is not sufficient for a definite diagnosis of NMS, which should be determined from the presence of other clinical symptoms, such as muscle rigidity, autonomic disturbance, and mental status changes. Physicians should be cognizant that muscle rigidity may appear after the serum $\mathrm{CK}$ level normalizes, as occurred in the present cases.

It is unclear why muscle rigidity appeared after the serum $\mathrm{CK}$ levels normalized in the present cases. This phenomenon indicates that the elevated CK levels are not due to muscle rigidity. It is also unclear how many patients with NMS show nonsimultaneous occurrence of $\mathrm{CK}$ elevation and muscle rigidity. Further studies are needed on the relationship between $\mathrm{CK}$ and degree of muscle rigidity during the course of NMS.

\section{Conclusion}

In general, it appears that both serum CK levels and muscle rigidity increase in the early stage of NMS, but there are exceptional cases, like those described here, where the elevated serum CK level and emergence of muscle rigidity do not appear in the same stage. In such cases, the diagnosis of NMS may be difficult and consequently the treatment may be delayed. While these NMS cases may be rare, physicians should keep in mind the possibility of exceptional NMS cases.

\section{Disclosure}

The author reports no conflicts of interest in this work.

\section{References}

1. Levenson JL. Neuroleptic malignant syndrome. Am J Psychiatry. 1985;142:1137-1145

2. Pope HG, Keck PE, McElroy SI. Frequency and presentation of neuroleptic malignant syndrome in a large psychiatric hospital. Am J Psychiatry. 1986;143:1227-1233.

3. Caroff SN, Mann SC. Neuroleptic malignant syndrome. Med Clin North Am. 1993;77:185-202.

4. American Psychiatric Association. Medication-induced movement disorders: neuroleptic malignant syndrome. Diagnostic and Statistical Manual of Mental Disorders: DSM-IV-TR. Washington, DC: American Psychiatric Association, 2000.

5. O'Dwyer AM, Sheppard NP. The role of creatine kinase in the diagnosis of neuroleptic malignant syndrome. Psychol Med. 1993;23:323-326.

6. Addonizio G, Susman VL, Roth DS. Neuroleptic malignant syndrome: review and analysis of 115 cases. Biol Psychiatry. 1987;22: 1004-1020.

7. Pelonero AI, Levenson JL, Pandurangi AK. Neuroleptic malignant syndrome: a review. Psychiatr Serv. 1998;49:1163-1172.

8. Rosebush P, Stewart T. A prospective analysis of 24 episodes of neuroleptic malignant syndrome. Am J Psychiatry. 1989;146:717-725.

9. Velamoor VR, Norman RMG, Caroff SN, Mann SC, Sullivan KA, Antelo RE. Progression of symptoms in neuroleptic malignant syndrome. J Nerv Ment Dis. 1994;182:168-173.

10. Sachdev PS. A rating scale for neuroleptic malignant syndrome. Psychiatry Res. 2005;135:249-256.

11. Suparivala A, Kant G, Jean RE. Neuroleptic malignant syndrome with metoclopramide overdose coexisting with Clostridium difficile diarrhea. Intensive Care Med. 2011;37:1706-1708.

12. Védie C, Poinso F, Hemmi F, Rivet B. Major symptoms and differential diagnosis of neuroleptic malignant syndrome: three case reports. Eur Psychiatry. 2000;15:334-337.

13. Harsch HH. Neuroleptic malignant syndrome: physiological and laboratory findings in a series of nine cases. J Clin Psychiatry. 1987;48: $328-333$.
Neuropsychiatric Disease and Treatment

\section{Publish your work in this journal}

Neuropsychiatric Disease and Treatment is an international, peerreviewed journal of clinical therapeutics and pharmacology focusing on concise rapid reporting of clinical or pre-clinical studies on a range of neuropsychiatric and neurological disorders. This journal is indexed on PubMed Central, the 'PsycINFO' database and CAS.

\section{Dovepress}

The manuscript management system is completely online and includes a very quick and fair peer-review system, which is all easy to use. Visit http://www.dovepress.com/testimonials.php to read real quotes from published authors. 